Acta Crystallographica Section D

Biological Crystallography

ISSN 0907-4449

\section{Marius Schmidt, ${ }^{\text {a* }}$ Vukica Srajer, ${ }^{\text {b }}$ Robert Henning, Hyotcherl thee, ${ }^{c, d}$ Namrta Purwar, ${ }^{\mathrm{a}}$ Jason Tenboer $^{\mathrm{a}}$ and Shailesh Tripathi ${ }^{a}$}

aPhysics Department, University of WisconsinMilwaukee, Milwaukee, Wisconsin, USA,

${ }^{\mathbf{b}}$ Center for Advanced Radiation Sources, The University of Chicago, Chicago, Illinois, USA, 'Center for Nanomaterials and Chemical Reactions, Institute for Basic Science, Daejeon 305-701, Republic of Korea, and dDepartment of Chemistry, KAIST, Daejeon 305-701, Republic of Korea

Correspondence e-mail: m-schmidt@uwm.edu

\title{
Protein energy landscapes determined by five-dimensional crystallography
}

Free-energy landscapes decisively determine the progress of enzymatically catalyzed reactions [Cornish-Bowden (2012), Fundamentals of Enzyme Kinetics, 4th ed.]. Time-resolved macromolecular crystallography unifies transient-state kinetics with structure determination [Moffat (2001), Chem. Rev. 101, 1569-1581; Schmidt et al. (2005), Methods Mol. Biol. 305, 115154; Schmidt (2008), Ultrashort Laser Pulses in Medicine and Biology] because both can be determined from the same set of $\mathrm{X}$-ray data. Here, it is demonstrated how barriers of activation can be determined solely from five-dimensional crystallography, where in addition to space and time, temperature is a variable as well [Schmidt et al. (2010), Acta Cryst. A66, 198206]. Directly linking molecular structures with barriers of activation between them allows insight into the structural nature of the barrier to be gained. Comprehensive time series of crystallographic data at 14 different temperature settings were analyzed and the entropy and enthalpy contributions to the barriers of activation were determined. One hundred years after the discovery of X-ray scattering, these results advance $\mathrm{X}$-ray structure determination to a new frontier: the determination of energy landscapes.

\section{Introduction}

Since the 1890s, the Van't Hoff-Arrhenius equation, $\nu \exp \left(-\beta E_{\mathrm{a}}\right)$, has been used to describe the temperature dependence of chemical reaction rates. $E_{\mathrm{a}}$ is the energy of activation and the factor $\beta=1 /\left(k_{\mathrm{B}} T\right)$ containing the Boltzmann factor $k_{\mathrm{B}}$ accounts for the inverse temperature behavior. The pre-factor $v$ accounts for the dynamic behavior of the members of the ensemble. Eyring (1935) tied this equation to a transition state at the top of the barrier of activation,

$$
k=\left(\frac{R T}{N_{\mathrm{A}} h}\right) \exp \left(\frac{\Delta S^{\#}}{R}\right) \exp \left(-\frac{\Delta H^{\#}}{R T}\right),
$$

where $R$ is the gas constant, $N_{\mathrm{A}}$ is Avogadro's number, $h$ is Planck's constant and $\Delta S^{\#}$ and $\Delta H^{\#}$ are the entropy and enthalpy differences from the initial state to the transition state, respectively. A reaction can be followed with timeresolved methods, from which conclusions on the underlying mechanism are drawn by kinetic modeling. In early approaches (Gibson, 1952; Austin et al., 1975), the structures of the reaction intermediates were inferred from static crystallography. Time-resolved crystallography (TRX; Moffat, 1989) finally unified kinetics with structure determination (Šrajer et al., 1996; Schmidt et al., 2003; Schmidt, 2008). Once the structures of intermediates are known, kinetic mechanisms can be tested by post-refinement against the TRX data (Schmidt, 2008; Schmidt et al., 2004). If the temperature is
Received 26 July 2013

Accepted 19 September 2013 
varied, the previously four-dimensional crystallographic data become five-dimensional (Schmidt et al., 2010). The photocycle of photoactive yellow protein (PYP) is used here as a model system from which five-dimensional crystallographic data were collected. The photocycle features distinct intermediate states, structures of which were determined earlier by picosecond and nanosecond TRX at only one temperature (Schotte et al., 2012; Jung et al., 2013; Ihee et al., 2005; Schmidt et al., 2004). Absorption of a blue photon at $485 \mathrm{~nm}$ provides $245 \mathrm{~kJ} \mathrm{~mol}^{-1}$ of energy to excite the central $p$-coumaric acid (pCA) chromophore (Fig. 1). Part of the energy is rapidly dissipated (Martin et al., 1983; Fitzpatrick et al., 2012). The remaining energy is stored in an energy-rich atomic configuration (Groenhof et al., 2004) labeled $\mathrm{I}_{\mathrm{T}}$. The chromophore is not yet fully isomerized from trans to cis (Schotte et al., 2012; van Stokkum et al., 2004; Jung et al., 2013). The $\mathrm{I}_{\mathrm{T}}$ state is followed by two states: $\mathrm{I}_{\mathrm{CT}}$ and $\mathrm{pR}_{1}$. $\mathrm{I}_{\mathrm{CT}}$ and $\mathrm{pR}_{1}$ are fully cis and branch away from $\mathrm{I}_{\mathrm{T}}$ in a volume-conserving bicycle-pedal and hula-twist reaction, respectively (Jung et al., 2013). The dominant species is $\mathrm{I}_{\mathrm{CT}}$. $\mathrm{In} \mathrm{I}_{\mathrm{CT}}$ the carbonyl $\mathrm{O}$ atom is flipped to the other side but the chromophore head is still fixed by two hydrogen bonds to amino acids Tyr42 and Glu46. In $\mathrm{pR}_{1}$ the chromophore head hydroxyl has lost one hydrogen bond. The entire chromophore has rotated about the chromophore axis.

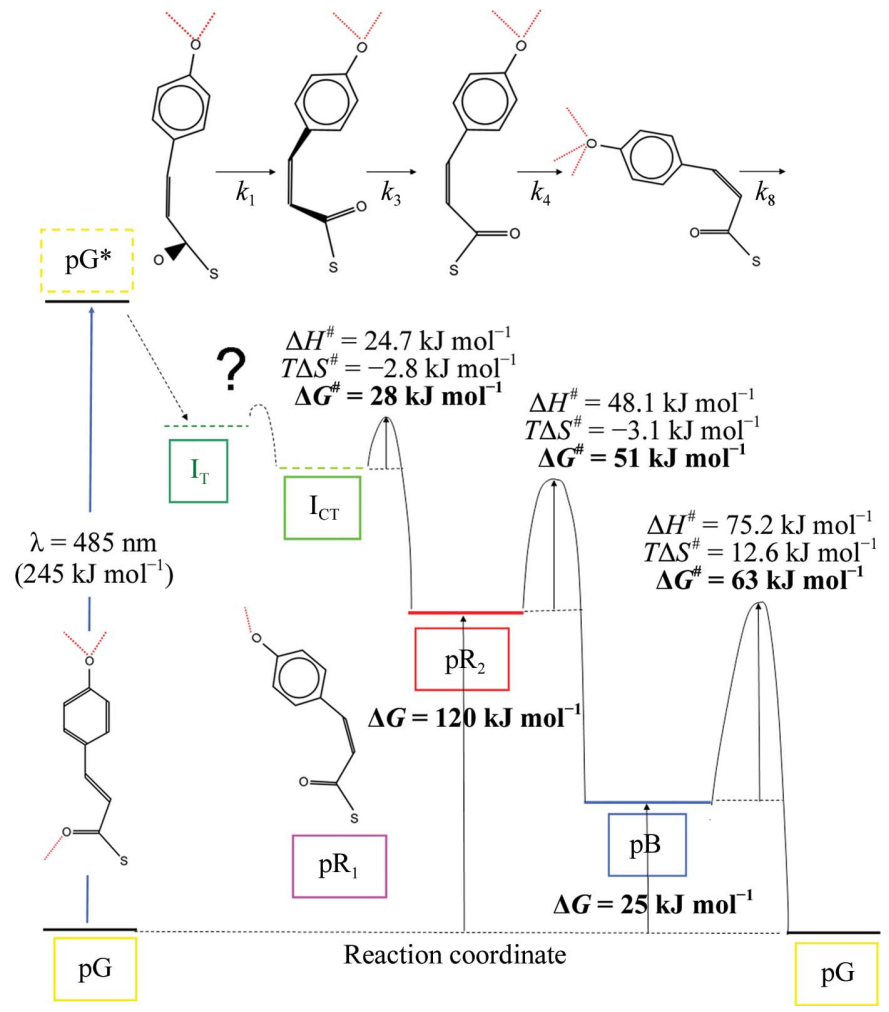

Figure 1

Free-energy landscape of the main reaction pathway in PYP. The reaction coordinate has periodic boundaries $(\mathrm{pG})$. Atomic structures of $\mathrm{pG}, \mathrm{I}_{\mathrm{T}}, \mathrm{I}_{\mathrm{CT}}$, $\mathrm{pR}_{1}, \mathrm{pR}_{2}$ and $\mathrm{pB}$ are known. Early transitions from $\mathrm{pG}^{*}$ require higher time resolution. The energy levels of $\mathrm{I}_{\mathrm{T}}$ and $\mathrm{I}_{\mathrm{CT}}$ are unknown (dashed lines); the free-energy levels of $\mathrm{pR}$ and $\mathrm{pB}$ are known in solution (Takeshita et al., 2002; van Brederode et al., 1995). The free-energy and entropic and enthalpic contributions to the barriers as extracted from the five-dimensional crystallographic data are shown at $300 \mathrm{~K}$.
$\mathrm{I}_{\mathrm{CT}}$ relaxes to $\mathrm{pR}_{2}$. This relaxation causes the Cys69 $\mathrm{S}$ atom to which the chromophore is bound to move significantly. The strongest difference electron-density features are found near this $\mathrm{S}$ atom (Fig. $2 b$ ). The $\mathrm{pR}_{1}$ and $\mathrm{pR}_{2}$ states are occupied for many orders of magnitude in time. Finally, they relax to the $\mathrm{pB}$ state (Ihee et al., 2005; Schmidt et al., 2004). The pB state most likely resembles the signaling state of PYP. The chromophore head forms new hydrogen bonds to the displaced Asp52 and to an additional water that appears near the entrance to the chromophore pocket (Tripathi et al., 2012). Finally, pB relaxes to the dark state $(\mathrm{pG})$. Microscopic rate coefficients $k$ between the intermediates plus the extent of reaction initiation specify a mechanism. The mechanism proposed by two previous TR crystallographic studies of PYP (Jung et al., 2013; Ihee et al., $2005)$ is depicted in Fig. 3. The rate coefficients of this mechanism depend on the temperature. This dependence can be described by the transition-state equation (TSE; equation 1). Other equations such as Kramer's equation (Hanggi et al., 1990), which parameterizes the pre-factors of the rate coefficients in terms of friction, are also frequently used. With this, our results would not be comparable with earlier results on PYP (Van Brederode et al., 1995, 1996; Ng et al., 1995), which were based on the TSE. Accordingly, we also use the TSE and express the barrier height in terms of enthalpy and entropy differences from the transition state. We demonstrate here how these thermodynamic parameters can be extracted solely from five-dimensional crystallography.

\section{Methods}

TRX experiments were conducted on crystals of PYP on the BioCARS 14-ID beamline at the Advanced Photon Source, Argonne National Laboratory, USA. Nanosecond laser pulses ( $\sim 5$ ns pulse duration) from a tunable Opolette HEII laser (Opotek) were used to initiate the photocycle and the reaction was followed from nanoseconds to seconds using X-ray pulses of $\sim 100 \mathrm{ps}$ duration. The temperature was varied from 233 to $343 \mathrm{~K}$ using a Cryojet (Agilent Technologies) up to $293 \mathrm{~K}$ and a Cryostream (Oxford Cryosystems) for higher temperatures. PYP crystals were grown as described elsewhere (Borgstahl et al., 1995). The PYP crystals were mounted in $1 \mathrm{~mm}$ glass capillaries glued into brass pins. For temperatures of $>303 \mathrm{~K}$ the capillaries must be insulated from the cold brass pins, since otherwise severe distillation effects would destroy the crystals (Fig. 4a). With this setup, temperatures of up to $343 \mathrm{~K}$ can be tolerated. The temperature at the crystal site was determined with a calibrated diode and used for subsequent calculations (Table 1). At $T>348 \mathrm{~K}$ wild-type PYP thermally denatures (Meyer et al., 2003) and, accordingly, the crystals start to decompose. Within an $\sim 110 \mathrm{~K}$ temperature range (233$343 \mathrm{~K}$ ), the photocycle was probed at 14 different temperature settings using 21-31 time points at each temperature (Table 1 and Supplementary Material ${ }^{\mathbf{1}}$ ). The Laue data were collected with the time as the fast variable: for a certain fixed crystal

\footnotetext{
${ }^{1}$ Supplementary material has been deposited in the IUCr electronic archive (Reference: DW5067).
} 


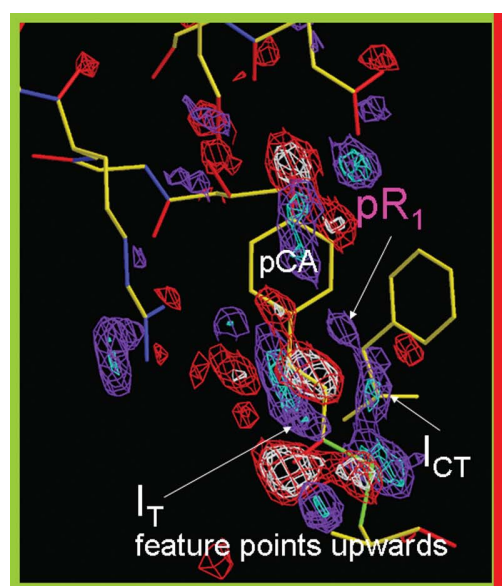

(a)

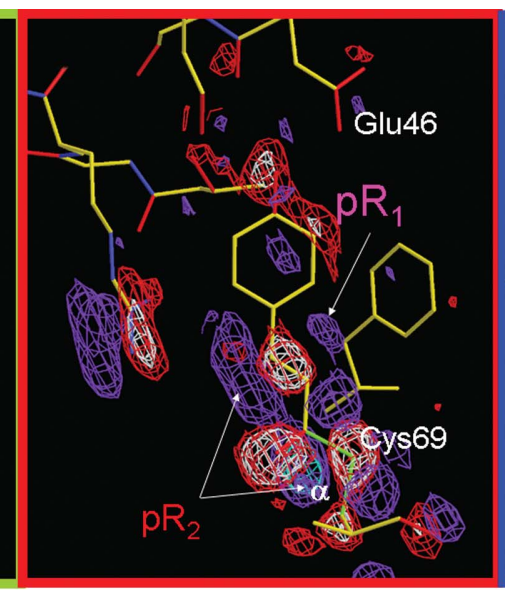

(b)

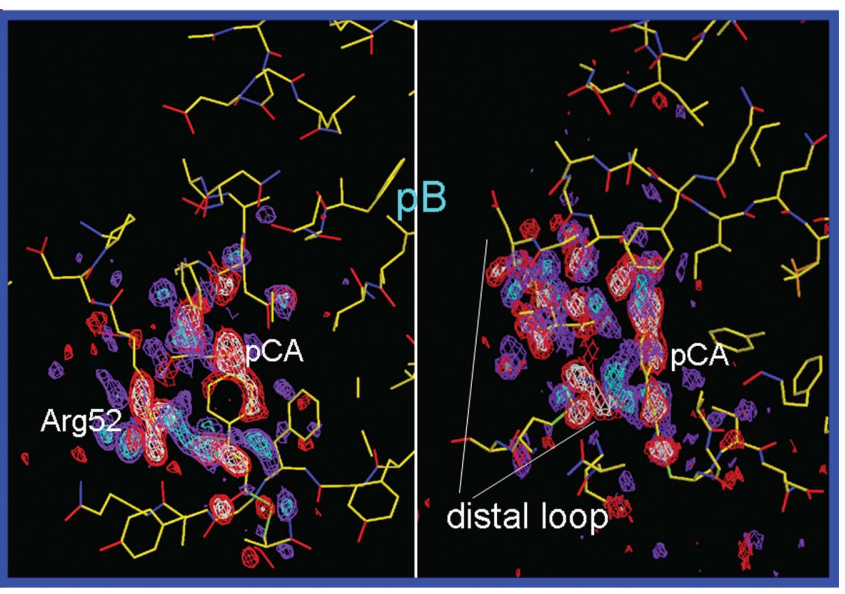

(c)

Figure 2

Experimental difference maps near the p-coumaric acid (pCA) chromophore in PYP averaged through various time intervals and at various temperatures and contoured at $\pm 3 \sigma$ in blue and red and $\pm 4 \sigma$ in cyan and white, respectively. Averaging was performed solely to enhance the appearances of the maps and to illustrate signature features of the intermediate states. (a) Average difference map obtained from the eight earliest difference maps from 2 to $8 \mathrm{~ns}$ at $T<263 \mathrm{~K}$. Features of three intermediates, $\mathrm{I}_{\mathrm{T}}, \mathrm{I}_{\mathrm{CT}}$ and $\mathrm{pR}_{1}$, contribute to the same set of maps. (b) Average difference map obtained from 20 difference maps at $T<263 \mathrm{~K}$ from $256 \mathrm{~ns}$ to $64 \mu$ s. Features of two intermediates, $\mathrm{pR}_{1}$ and $\mathrm{pR}_{2}$, contribute. Feature $\alpha$ is the strongest in all maps at up to $12 \sigma$ above the noise. It denotes the displacement of the Cys69 S atom in $\mathrm{pR}_{2}$. (c) Average difference map obtained from 20 difference maps in the millisecond time range at $303<T<323 \mathrm{~K}$. Main features are found near the chromophore and on the distal loop.

orientation diffraction patterns for all time points were collected. After this, the crystal was set to another orientation and translated to expose a fresh crystal volume. The process was repeated until a complete data set had been collected. 20 different crystal orientations were used to cover reciprocal space. The data sets were processed by Precognition/Epinorm (RenzResearch; see Table 1 and Supplementary Material for data statistics). Difference electron-density maps were calculated on the absolute scale using weighted difference structure factors as described elsewhere (Tripathi et al., 2012; Schmidt, 2008). The time-dependent difference maps were analyzed

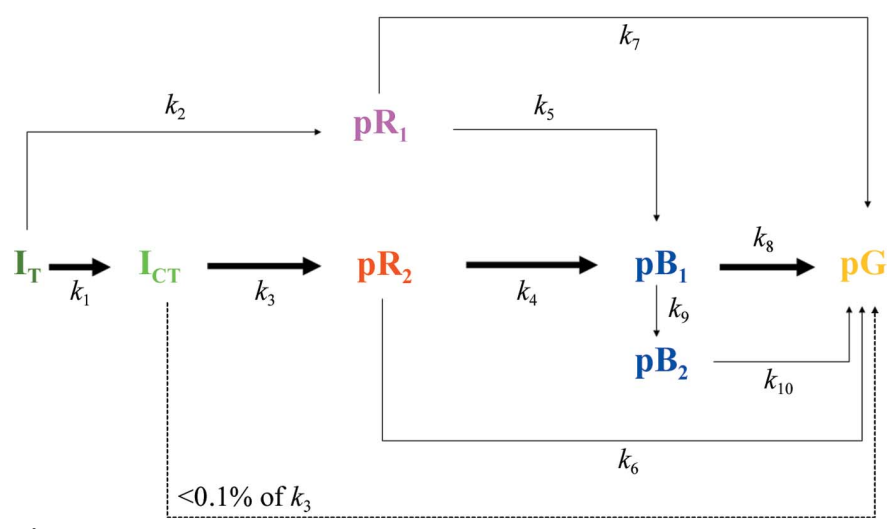

Figure 3

Chemical kinetic mechanisms and rate coefficients of the PYP photocycle. At temperatures up to $313 \mathrm{~K}$, eight rate coefficients $\left(k_{1}\right.$ to $k_{8}$ ) and five intermediate states plus the dark state contribute. The occupancy of $\mathrm{pB}_{2}$ is very low and cannot be observed. Above $313 \mathrm{~K}$ the early intermediates are not detected because the time series start around 100 ns. $\mathrm{pB}_{2}$ accumulates to a detectable extent and the rate coefficients $k_{9}$ and $k_{10}$ contribute in addition. The main reaction pathway in PYP is indicated by bold arrows. The direct path from $\mathrm{I}_{\mathrm{CT}}$ to $\mathrm{pG}$ is irrelevant (dashed arrow). by singular value decomposition (SVD) using the program SVD4TX (Schmidt et al., 2003; Schmidt, 2008). For the SVD, the difference maps of a time series were arranged in temporal order in data matrix A (see Supplementary Material for further details). Matrix A was subsequently decomposed into left singular vectors (ISVs) in matrix $\mathbf{U}$, representing spatial components, and right singular vectors (rSVs), which contain the temporal information of the corresponding $1 \mathrm{SVs}$, in matrix $\mathbf{V}$ (2). The diagonal matrix $\mathbf{S}$ contains the singular values.

$$
\mathbf{A}=\mathbf{U S V}^{T}
$$

The significant rSVs at each temperature can easily be identified (see $\S 3$ ). All $j$ significant rSVs of a time series were fitted globally by sums of exponentials, employing a common set of observable kinetic rates $\Lambda_{i}$. Different sets of amplitudes $A_{i, j}$ plus offsets $A_{0, j}$ were used for each individual rSV (3),

$$
\mathrm{rSV}_{j}=A_{0, j}+\sum_{i, j} A_{i, j} \exp \left(-\Lambda_{i} t\right)
$$

The relaxation times $\tau_{i}$ of the kinetic phases in the reaction are the reciprocals of $\Lambda_{i}$. The analysis was performed for each temperature, resulting in a set of temperature-dependent observable rates and corresponding relaxation times.

To determine microscopic rate coefficients of the individual interconversions between the intermediates, kinetic modeling of the five-dimensional crystallographic data is necessary. This is accomplished using (4) and (5). The mechanism displayed in Fig. 3 was employed. Time-dependent fractional concentrations of the intermediates, $c_{i}(t, k)$, were calculated by the program GetMech (Schmidt et al., 2004) by integrating the coupled differential equations (Steinfeld et al., 1985) that describe this mechanism. The concentrations were used to generate time-dependent difference maps from the time- 
Table 1

Statistics for selected Laue data sets at selected temperatures (for all temperatures, see Supplementary Material).

$T_{\text {set }}$ is the temperature set by the temperature controller of the cryogenic gas jet. $T_{\mathrm{c}}$ is the actual temperature measured by the calibrated diode at the crystal site. The time delays shown are the delays between the peak of the laser pulse to the rising edge of the X-ray pulse. The completeness of the Laue data was calculated including singlet and deconvoluted harmonic reflections. $R_{\text {merge }}$ is calculated from singlet intensities using multiple measurements and symmetry equivalents. Both completeness and $R_{\text {merge }}$ are given for the dark data set (the statistics are comparable for light data sets). Values in parentheses are for the last resolution shell (1.9-1.8 $\AA$ ). $R_{\text {scale }}$ is calculated from amplitudes $(F)$ after scaling the timeresolved structure-factor amplitudes $F^{\Delta t}$ to calculated dark $F^{\mathrm{D}}$ amplitudes (on the absolute scale). $\Delta \rho_{\min } / \sigma_{\Delta \rho}$ and $\Delta \rho_{\max } / \sigma_{\Delta \rho}$ are the most negative and most positive difference electron-density features in units of the $\sigma$ level found in the difference map at a selected time point $(\Delta t)$. The largest features can be found at and near the S atom of Cys69.

\begin{tabular}{llllllll}
\hline $\begin{array}{l}T_{\text {set }} \\
(\mathrm{K})\end{array}$ & $\begin{array}{l}T_{\mathrm{c}} \\
(\mathrm{K})\end{array}$ & Time points & Completeness (\%) & $I / \sigma(I)$ & $\begin{array}{l}R_{\text {merge }} \dagger \\
(\%)\end{array}$ & $\begin{array}{l}R_{\text {scale }}, \Delta t \ddagger \\
(\%)\end{array}$ & $\begin{array}{l}\Delta \rho_{\min } / \sigma_{\Delta \rho} \text { and } \\
\Delta \rho_{\text {max }} / \sigma_{\Delta \rho}, \Delta t\end{array}$ \\
\hline 233 & 235.5 & $31,2 \mathrm{~ns}-15 \mathrm{~s}$ & $82.5(75.9)$ & $16.9(11.0)$ & 8.3 & $7.7,3 \mu \mathrm{s}$ & $-6 /+10,3 \mu \mathrm{s}$ \\
263 & 259.5 & $29,2 \mathrm{~ns}-8 \mathrm{~s}$ & $88.0(82.3)$ & $23.0(18.5)$ & 6.8 & $6.9,4 \mu \mathrm{s}$ & $-7 /+9,4 \mu \mathrm{s}$ \\
283 & 283.6 & $27,2 \mathrm{~ns}-128 \mathrm{~ms}$ & $82.2(77.8)$ & $30.3(26.9)$ & 5.2 & $3.9,4 \mu \mathrm{s}$ & $-10 /+11,4 \mu \mathrm{s}$ \\
303 & 298.5 & $27,2 \mathrm{~ns}-128 \mathrm{~ms}$ & $83.5(75.9)$ & $25.3(16.1)$ & 5.6 & $6.8,4 \mu \mathrm{s}$ & $-11 /+10,4 \mu \mathrm{s}$ \\
343 & 338.8 & $21,100 \mathrm{~ns}-1 \mathrm{~s}$ & $84.6(80.3)$ & $30.6(21.7)$ & 4.7 & $4.4,800 \mathrm{~ns}$ & $-6 /+6,800 \mathrm{~ns}$ \\
\hline
\end{tabular}

$\dagger R_{\text {merge }}=\sum_{h k l} \sum_{i}\left|I_{i}(h k l)-\langle I(h k l)\rangle\right| / \sum_{h k l} \sum_{i} I_{i}(h k l) . \ddagger R_{\text {scale }}=\sum_{h k l}\left|F_{h k l}^{\Delta t}-F_{h k l}^{\mathrm{D}}\right| / \sum_{h k l} F_{h k l}^{\mathrm{D}}$.

2002). The nomenclature used in previous studies (Genick et al., 1997; Ihee et al., 2005; Kim et al., 2012; Yeremenko et al., 2006; Jung et al., 2013) is followed here. The structures of the intermediates are, in order of appearance after reaction initiation (Fig. 3), $\mathrm{I}_{\mathrm{T}}, \mathrm{pR}_{1}, \mathrm{I}_{\mathrm{CT}}, \mathrm{pR}_{2}, \mathrm{pB}_{1}$ and $\mathrm{pB}_{2}$ (Ihee et al., 2005; Jung et al., 2013), with PDB entries 3ve3, 1ts7, 3ve4, 1ts0 and 1ts6, respectively. The structure of the dark/reference state was obtained from PDB entry 2phy (Borgstahl et al., 1995). It was assumed here that the structural differences between the intermediates and the dark structure are temperatureindependent. The program GetMech was further used to optimize the microscopic rate coefficients, $k$, of the assumed mechanism (Fig. 3). By varying the microscopic rate coefficients, the

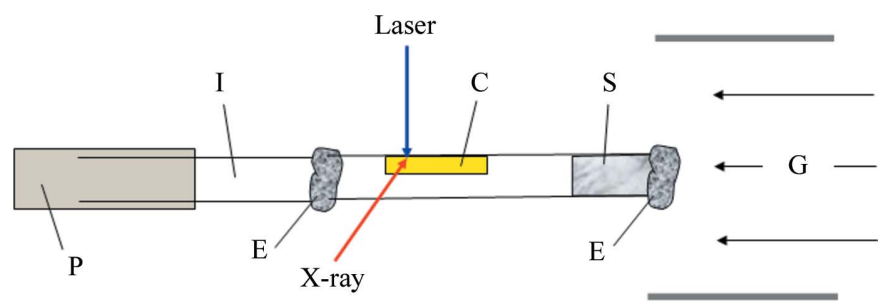

(a)

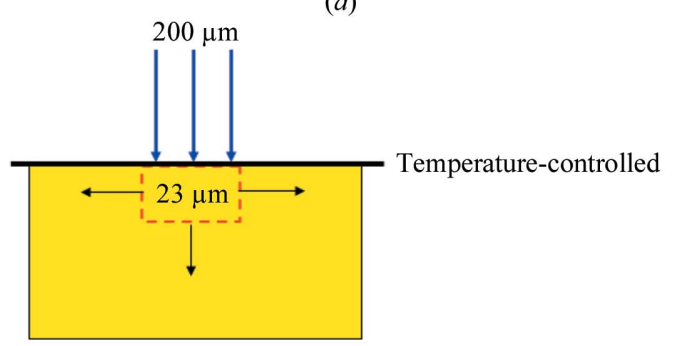

(b)

\section{Figure 4}

Crystal capillary mount to accommodate temperatures of $>303 \mathrm{~K}$. (a) P, brass pin; I, insulating glass capillary; E, epoxy glue; C, PYP crystal; S, stabilizing solution; G, temperature-controlled gas stream. (b) Laserilluminated volume of the crystal (red dashed box). The capillary wall (thick black line) is temperature-controlled. Black arrows: heat diffuses out.

independent difference maps of the intermediates according to

$$
\Delta \rho(t)^{\mathrm{calc}}=\sum_{i=1}^{N} c_{i}(t, k) \Delta \rho_{i}^{\mathrm{ind}} .
$$

The time-independent difference maps of the intermediates $\Delta \rho_{i}^{\text {ind }}$ used in (4) were calculated by subtracting the structure factors of the dark-state structure from those of the respective (known) intermediate structures and subsequent Fourier synthesis. The structures of the dark state and intermediates were obtained from the Protein Data Bank (Berman et al., concentrations $c_{i}(t, k)$ are modified, which subsequently change the calculated time-dependent difference maps $\Delta \rho(t)^{\text {calc }}$ via (4). These calculated time-dependent difference maps were compared with all measured (observed) difference maps $\Delta \rho(t)^{\text {obs }}$ for each particular time series (5) and the rate coefficients were varied until convergence (Schmidt, 2008; Tripathi et al., 2012; Schmidt et al., 2012),

$$
\sum_{t}\left[\Delta \rho_{t}^{\text {obs }}-\mathrm{sf} \cdot \Delta \rho_{t}^{\text {calc }}(k)\right]^{2} \rightarrow \min .
$$

In addition, a scale factor ( $\mathrm{sf}$ ) is determined by (5) that is equal to the extent of reaction initiation. The extent of reaction initiation is equivalent to the fractional concentration of PYP molecules that are active (not in the dark state) in the crystal at the beginning of our time series. As a result, temperaturedependent microscopic rate coefficients were obtained. These were then fitted by the TSE to obtain the entropy and enthalpy differences of the barriers of activation. The programs SVD $4 T X$ and GetMech can be found on the web page of MS (http://users.physik.tu-muenchen.de/marius/ Software.htm).

Time-resolved absorption spectra were collected from crushed PYP crystals following reaction initiation at time delays from $20 \mu$ s to a few seconds at 273 and $303 \mathrm{~K}$ with a home-built fast microspectrophotometer (Purwar et al., 2013). Time-dependent difference absorption spectra were obtained by subtracting the time-resolved absorption spectra from that obtained from PYP in the dark. The time series were also analyzed by SVD using MatLab (MathWorks) routines to extract relaxation times (see Supplementary Material for further details).

\section{Results}

Table 1 gives an overview of the collected Laue data (also see Supplementary Material). Since the recent upgrade of the 
BioCARS 14-ID beamline (Graber et al., 2011), Laue data of excellent quality with $R_{\text {merge }}$ in the range of $5 \%$ and $I / \sigma(I)>20$ can be collected rapidly (Tripathi et al., 2012; Schmidt et al., 2012), which makes comprehensive studies such as this possible. Fig. 5(a) shows an example of right singular vectors (rSVs) extracted from the TRX data by SVD (Schmidt et al., 2003). The right singular values are exquisitely smooth owing to the excellent data quality and data-collection strategies at BioCARS (Graber et al., 2011). As shown previously (Schmidt et al., 2012), an entire time series can be collected from a single PYP crystal; the absorbed X-ray dose and damage inflicted by the laser is below the kinetic dose limit $D_{\mathrm{K}}^{1 / 2}$ for PYP. In this way, the crystal-to-crystal scaling variations which plagued earlier investigations (Ihee et al., 2005; Rajagopal et al., 2005; Schmidt, Nienhaus et al., 2005) are avoided. As a result, although rSVs 1-15 are displayed in Fig. 5(a), the less significant rSVs (4-15) are distributed closely around zero (colored thin lines in Fig. 5a). A global fit with four exponentials identifies four kinetic processes $\Lambda_{1} \ldots \Lambda_{4}$ with relaxation times $\tau_{1} \ldots \tau_{4}$. The process with relaxation time $\tau_{1}$ results from the nonzero laser pulse width and the decay of $\mathrm{I}_{\mathrm{T}}$ to both $\mathrm{I}_{\mathrm{CT}}$ and $\mathrm{pR}_{1}$ that can be identified in the earliest difference maps. Processes $\tau_{2}$ to $\tau_{4}$ result from relaxations of states $\mathrm{I}_{\mathrm{CT}}$ to $\mathrm{pR}$ $\left(\tau_{2}\right)$, the joint relaxations of $\mathrm{pR}_{1}$ and $\mathrm{pR}_{2}$ to $\mathrm{pB}$ and $\mathrm{pG}\left(\tau_{3}\right)$ and finally from $\mathrm{pB}$ to $\mathrm{pG}\left(\tau_{4}\right)$, respectively. These processes accelerate when the temperature is increased (see Fig. $5 a$, numbers shown in red). The rSVs as well as the relaxation times at all temperatures can be found in Supplementary Fig. S3. In Figs. $5(b)-5(d)$, the relaxation rates $\Lambda_{\mathrm{i}}$ are plotted as a function of temperature and fitted by the Van't HoffArrhenius equation (dashed lines). The pre-factors and the energies of activation $E_{\mathrm{a}}$ derived from the fits are shown in Table 2. Slower relaxation times from TRX and time-resolved microspectroscopy (TRS) agree reasonably (Fig. 6 and Table 2). At $273 \mathrm{~K}$ three processes are observed in TRS. Process (1) corresponds to the $\mathrm{pR}$ to $\mathrm{pB}$ transition. The relaxation time derived from TRS is $2.1 \mathrm{~ms}$, which compares with process $\tau_{3}(0.7 \mathrm{~ms})$ obtained from $\mathrm{TRX}$. The $\mathrm{pB}$ to $\mathrm{pG}$ transition is biphasic (processes 2 and 3 ). The relaxation time of process (2) is $67 \mathrm{~ms}$ at $273 \mathrm{~K}$, compared with $\tau_{4}=73 \mathrm{~ms}$ obtained from TRX, and $2.2 \mathrm{~ms}$ at $303 \mathrm{~K}$, compared with $\tau_{4}=$ $6 \mathrm{~ms}$ observed crystallographically. Process (3) observed with

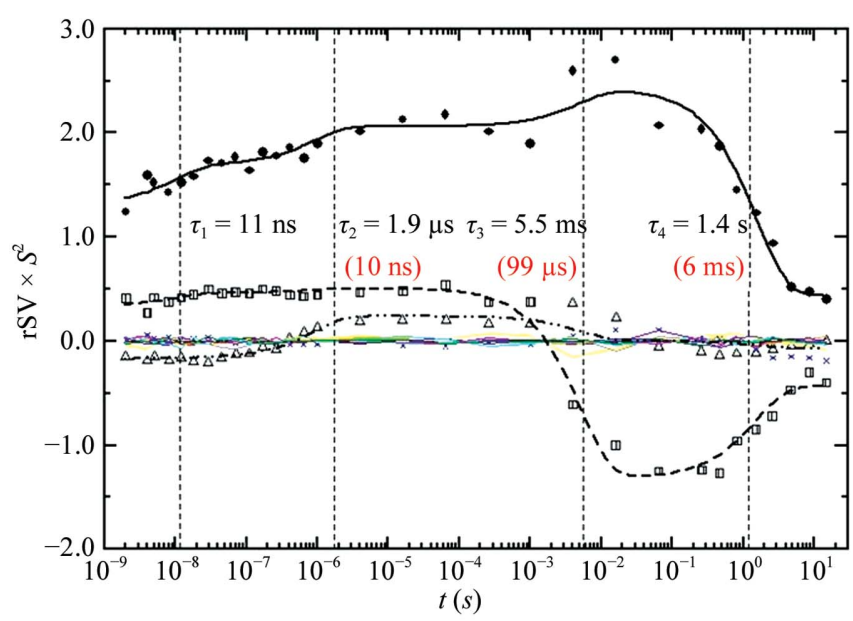

(a)

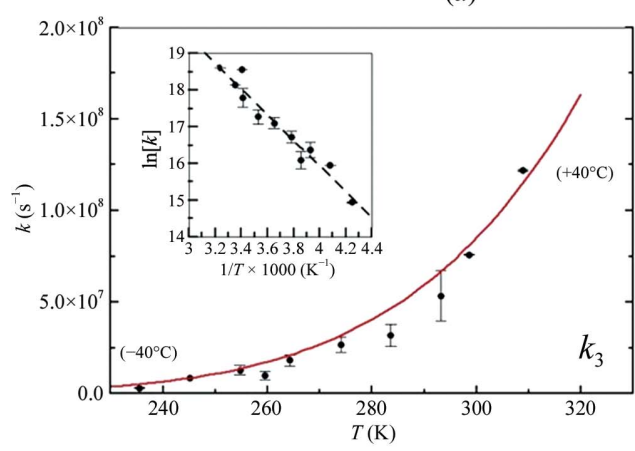

(e)

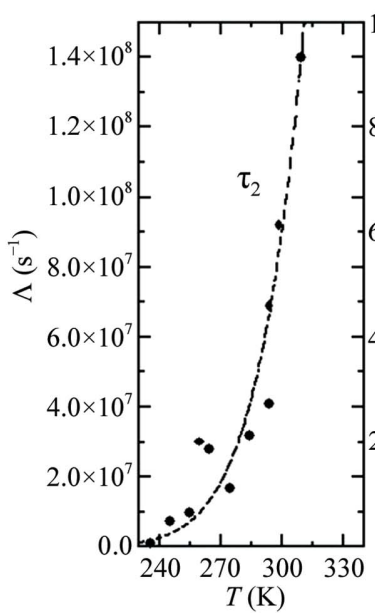

(b)

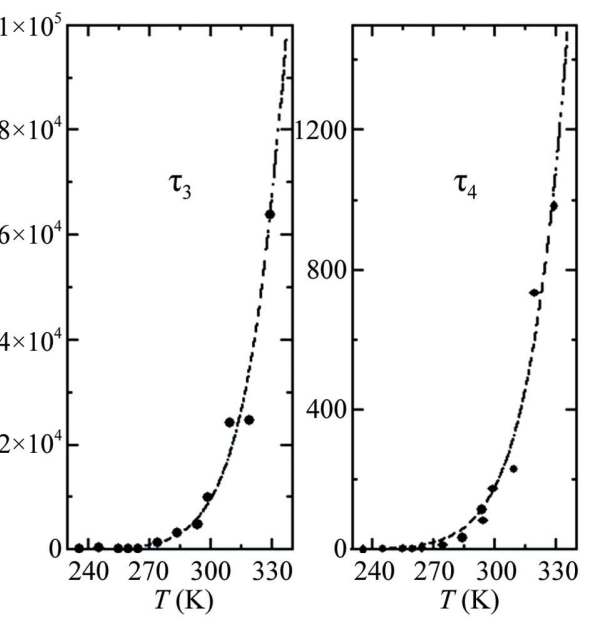

(c)

(d)

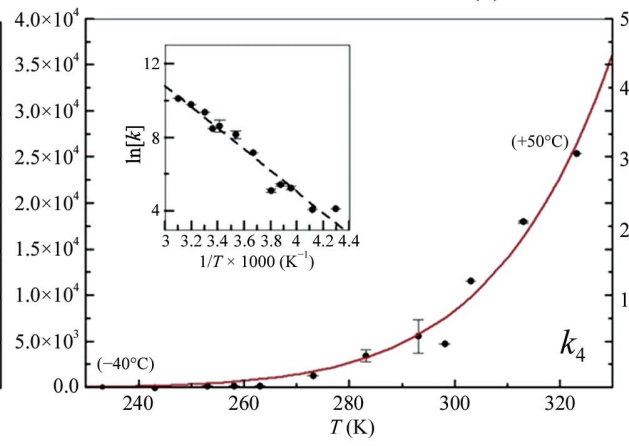

$(f)$

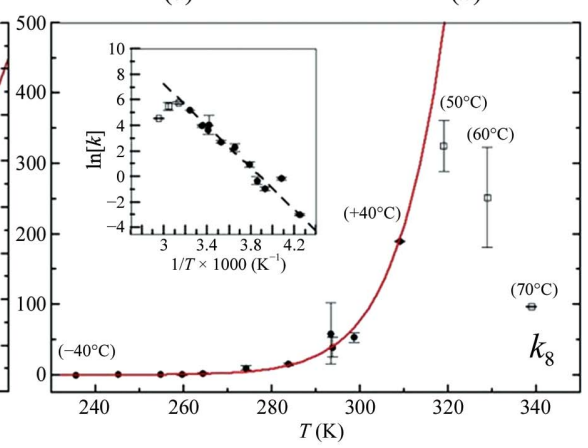

$(g)$

Figure 5

(a) Right singular vectors (rSVs) at $233 \mathrm{~K}$. The rSVs are weighted by the square of their respective singular value $S$. Four kinetic processes $\tau_{1} \ldots \tau_{4}$ are globally observed (dashed vertical lines). Solid spheres, open triangles and squares: first, second and third significant rSVs. Colored thin lines around zero: remaining less significant rSVs. Solid black line, dashed line and dashed double dotted line: global fit of the significant rSVs by four exponential functions with the same set of relaxation times but different amplitudes. Relaxation times obtained at room temperature (298 K, red) are shown in parentheses for comparison. $(b),(c)$ and $(d)$ : macroscopic rates $\Lambda$ (inverse of relaxation times) for processes $\tau_{2}$, $\tau_{3}$ and $\tau_{4}$ plotted as a function of temperature, respectively. Dashed lines: fits by the Van't Hoff-Arrhenius equation. $(e),(f)$ and $(g)$ : temperature dependence of the main pathway microscopic rate coefficients $k_{3}, k_{4}$ and $k_{8}$, respectively. Red lines, fits by the TSE. Insets, Arrhenius plots: dashed lines, fits by straight lines. 
Table 2

Energetics of the PYP photocycle.

(a) Macroscopic observable rates $\Lambda_{i}$ from TR crystallography. Temperature dependences are fitted by the Van't Hoff-Arrhenius equation. The temperature dependence of process $\tau_{1}\left(\Lambda_{1}\right)$ cannot be determined owing to limited time resolution. n.a., not applicable.

\begin{tabular}{lllll}
\hline Macroscopic rate coefficients & $\Lambda_{1}$ & $\Lambda_{2}$ & $\Lambda_{3}$ & $\Lambda_{4}$ \\
\hline Pre-factor $v\left(\mathrm{~s}^{-1}\right)$ & n.a. & $1.6 \times 10^{14}$ & $1.9 \times 10^{13}$ & $7.7 \times 10^{10}$ \\
Energy of activation $E_{\mathrm{a}}\left(\mathrm{kJ} \mathrm{mol}^{-1}\right)$ & n.a. & 35.9 & 53.4 & 49.6 \\
\hline
\end{tabular}

(b) Energetics derived from fitting the TSE to the temperature dependence of selected microscopic rate coefficients (the errors from the fit are given in parentheses).

\begin{tabular}{lcrrl}
\hline Microscopic rate coefficients & \multicolumn{1}{c}{$k_{3} \dagger$} & \multicolumn{1}{c}{$k_{4} \dagger$} & \multicolumn{1}{c}{$k_{5} \dagger$} & \multicolumn{1}{l}{$k_{8}$} \\
\hline$\Delta H^{\#}\left(\mathrm{~kJ} \mathrm{~mol}^{-1}\right)$ & 24.7 & 48.1 & 50.0 & $75.2(0.03)$ \\
$\Delta S^{\#}\left(\mathrm{~J} \mathrm{~mol}^{-1} \mathrm{~K}^{-1}\right)$ & -9.4 & -10.2 & -14.8 & $41.9(0.08)$ \\
$T \Delta S^{\#} \ddagger\left(\mathrm{kJ} \mathrm{mol}^{-1}\right)$ & -2.8 & -3.1 & -4.5 & $12.6(0.02)$ \\
$\Delta G^{\#} \ddagger\left(\mathrm{kJ} \mathrm{mol}^{-1}\right)$ & 27.5 & 51.2 & 54.5 & $62.7(0.09)$ \\
\hline
\end{tabular}

(c) Comparison of processes (1) to (3) observed by TRS with those derived from TRX $\left(\tau_{3}\right.$ and $\left.\tau_{4}\right)$. An extra phase (3) is observed by TRX only at elevated temperatures. n.o., not observed.

\begin{tabular}{llll}
\hline Processes observed & $(1) / \tau_{3}$ & $(2) / \tau_{4}$ & $(3)$ \\
\hline $273 \mathrm{~K}$ & & & \\
TRS & $2.1 \mathrm{~ms}$ & $67 \mathrm{~ms}$ & $800 \mathrm{~ms}$ \\
TRX & $0.7 \mathrm{~ms}$ & $70 \mathrm{~ms}$ & n.o. \\
$303 \mathrm{~K}$ & & & \\
TRS & n.o. & $2.2 \mathrm{~ms}$ & $32 \mathrm{~ms}$ \\
TRX & $99 \mu \mathrm{s}$ & $6 \mathrm{~ms}$ & $40 \mathrm{~ms}(323 \mathrm{~K})$ \\
\hline
\end{tabular}

$\dagger$ The errors of the fitted parameters are smaller than $1 \%$. $末$ At $300 \mathrm{~K}$.

TRS at $273 \mathrm{~K}$ contributes in a minor way, so that it cannot be detected with TRX. However, at elevated temperatures it can also be detected by crystallography with a similar relaxation time. Notably, the photocycle can be observed crystallographically up to $343 \mathrm{~K}$ and PYP also remains active at low temperatures $(233 \mathrm{~K})$ where the photocycle completes in $\sim 10 \mathrm{~s}$.

The main reaction pathway through $\mathrm{pR}_{2}$ follows microscopic rate coefficients $k_{1}, k_{3}, k_{4}$ and $k_{8}$ (compare Figs. 1 and 3). A minor pathway involves $\mathrm{pR}_{1}$ which branches away from $\mathrm{I}_{\mathrm{T}}$. Rate coefficient $k_{7}$ is generally $50 \%$ of $k_{5}$. One third of the $\mathrm{pR}_{1}$ molecules relax directly to $\mathrm{pG}$, while two thirds populate $\mathrm{pB}$. Since the $\mathrm{pR}_{1}$ occupancy is low, the rate coefficient $k_{7}$ is difficult to determine and therefore may vary substantially. $\mathrm{pR}_{2}$ typically decays mainly to $\mathrm{pB} ; k_{6}$ is generally much smaller than $k_{4}$. In earlier PYP studies (Ihee et al., 2005; Tripathi et al., 2012) the pathways through $k_{6}$ and $k_{7}$ were not taken into account. Here we considered them as well as a more general possibility. Both pathways add little to the mechanism, since the $\mathrm{pR}_{1}$ occupancy is small and only a small fraction of $\mathrm{pR}_{2}$ relaxes directly to $\mathrm{pG}$. The main product is $\mathrm{pB}$, which decays with rate $k_{8}$. At higher temperatures only rate coefficients $k_{4}$ to $k_{10}$ were included. This mechanism lacks the early intermediates as their population decays rapidly compared with the earliest time delay. It features in addition two scale factors that account for the amount of $\mathrm{pR}_{1}$ and $\mathrm{pR}_{2}$ and an extra state $\mathrm{pB}_{2}$.
The two pR states are fully occupied after $20 \mathrm{~ns}$ at these temperatures (Jung et al., 2013) and relax to $\mathrm{pB}$ on the microsecond time scale. At $323 \mathrm{~K}$ a weak second $\mathrm{pB}$ phase appears that indicates the presence of $\mathrm{pB}_{2}$ (see Supplementary Fig. S3). Rather than speeding up, the photocycle slows down (Fig. 5g) because the PYP occupies additional pB-like states even in the crystal. The concentration profiles of the intermediates closely display the relaxation times observed in the rSVs (see also Supplementary Fig. S3).

For all rate coefficients, except for $k_{1}$ and $k_{2}$, where we are limited by the time resolution of the experiment, thermal activation is observed. The photocycle accelerates by a factor of 318 when the temperature is changed by $80 \mathrm{~K}$ (from $1.4 \mathrm{~s}$ at $233 \mathrm{~K}$ to $4.4 \mathrm{~ms}$ at $313 \mathrm{~K}$ ). This agrees nicely with the well known $Q_{10}$ rule, which predicts that the reaction velocity in enzymes doubles when the temperature increases by $10 \mathrm{~K}$. The $Q_{10}$ rule predicts an acceleration by a factor of $256\left(2^{8}\right)$ compared with the factor of 318 observed here. Above $323 \mathrm{~K}$ PYP surpasses its temperature optimum and the photocycle slows down again (Fig. $5 g$ ). Therefore, the rate coefficients extracted at 333 and $343 \mathrm{~K}$ were not included in the fit of the TSE. In Figs. $5(e)-5(g)$ the temperature dependences of $k_{3}$, $k_{4}$ and $k_{8}$ are shown (those of $k_{5}$ and $k_{7}$ are shown in the Supplementary Material). The barrier height varies from $27.5 \mathrm{~kJ} \mathrm{~mol}^{-1}$ for $\mathrm{I}_{\mathrm{CT}}$ decay $\left(k_{3}\right)$ to $63 \mathrm{~kJ} \mathrm{~mol}^{-1}$ for $\mathrm{pB}_{1}$ depopulation $\left(k_{8}\right)$ (Fig. 1 and Table 2). Absorption of the laser pulse deposits energy into a volume approximately determined by the laser footprint on the crystal, the crystal diameter and the penetration depth (Fig. 4b). An adiabatic temperature jump of about $11 \mathrm{~K}$ is estimated (see Supplementary Material). By correcting for the jump, the barriers shift by only $\sim 1.5 \mathrm{~kJ} \mathrm{~mol}^{-1}$ and the entropic contributions remain almost the same. A portion of the absorbed energy is initially stored in the twisted chromophore geometry of $\mathrm{I}_{T}$ and is only released gradually through exothermic relaxation processes. Owing to the shallow penetration depth of the laser, the heat dissipates rapidly on a submillisecond time scale. The final $\mathrm{pB}$ to $\mathrm{pG}$ relaxation is never affected. Accordingly, we report uncorrected values here.

\section{Discussion}

The mechanism employed (Fig. 3) was motivated by earlier TRX experiments with nanosecond (Ihee et al., 2005) and picosecond (Jung et al., 2013) time resolution using PYP crystals grown as originally described in Borgstahl et al. (1995). Another picosecond TRX experiment was recently performed (Schotte et al., 2012) on different PYP crystals which were grown in heavy water and at high salt concentrations (1.1 $\mathrm{M}$ $\mathrm{NaCl}, \mathrm{pD}$ 9) and that were intended to be used in neutron diffraction experiments. Between these two approaches, there are very subtle differences in the intermediate structures on fast time scales and two major differences on longer time scales. (i) The final relaxation time from $\mathrm{pB}$ to $\mathrm{pG}$ observed by Schotte and coworkers at $288 \mathrm{~K}$ is much longer ( $\sim 260 \mathrm{~ms}$ ) than that of $\sim 20 \mathrm{~ms}$ observed by Ihee and corworkers and also by us. (ii) Schotte and coworkers did not observe the inter- 
mediate that we call $\mathrm{pR}_{1}$ here and interpret their $10 \mathrm{~ns}-100 \mu \mathrm{s}$ time range using only a single $\mathrm{pR}_{2}$-like structure. Hence, the pathway through rate coefficients $k_{2}, k_{5}$ and $k_{7}$ (Fig. 3) was not included in their mechanism. This pathway must be added to our mechanism, since $\mathrm{pR}_{1}$ is observed in our difference maps at the earliest times (Fig. 2a) and continues to be occupied into the long microsecond time range until it decays simultaneously with $\mathrm{pR}_{2}$. The most likely reason for the differences in the detailed kinetic mechanism and altered relaxation times of Schotte and coworkers is that their PYP crystals were grown under quite different conditions (heavy water and high salt; see above). Even when the $\mathrm{pH}$ is shifted, a previous $\mathrm{pH}$ dependent TRX study with the same type of crystals that we used (Tripathi et al., 2012) shows that $\mathrm{pR}_{1}$ is present at all $\mathrm{pH}$ values ( $\mathrm{pH} \mathrm{4,7}$ and 9) and decays in concert with $\mathrm{pR}_{2}$. Since we knew that the external conditions such as the $\mathrm{pH}$ and salt concentration can alter the mechanism (Tripathi et al., 2012; Borucki et al., 2005, 2006), we used previously established uniform crystallization conditions throughout and we very carefully adjusted the $\mathrm{pH}$ to 7 for our study.

The TSE describes the temperature dependence of the rate coefficients $k$ in the range from 233 to $323 \mathrm{~K}$. This makes it possible to infer barriers of activation in the PYP photocycle in the crystal (Fig. 1). In Fig. 1 we adopted some of the conformational free energies from solution (Takeshita et al., 2002; van Brederode et al., 1995) with the strong caveat that they might be very different in the crystal. Barriers of activation from TRX are shown for the main reaction pathway. The final barrier is the rate-limiting step of the reaction. It slows down the photocycle decisively so that the signaling state may persist. The chromophore pocket opens to the solvent and becomes exceptionally susceptible to additional stimuli such as the $\mathrm{pH}$ (Tripathi et al., 2012; Borucki et al., 2006).

Fitting the Van't Hoff-Arrhenius equation to macroscopic rates, $\Lambda$, yields apparent energies of activation $E_{\mathrm{a}}$ of the

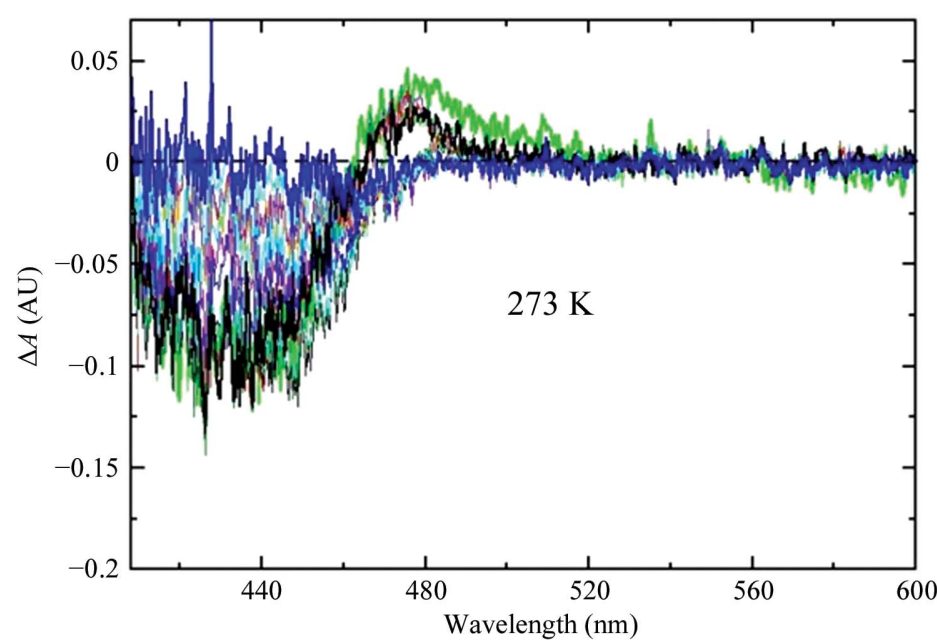

(a)

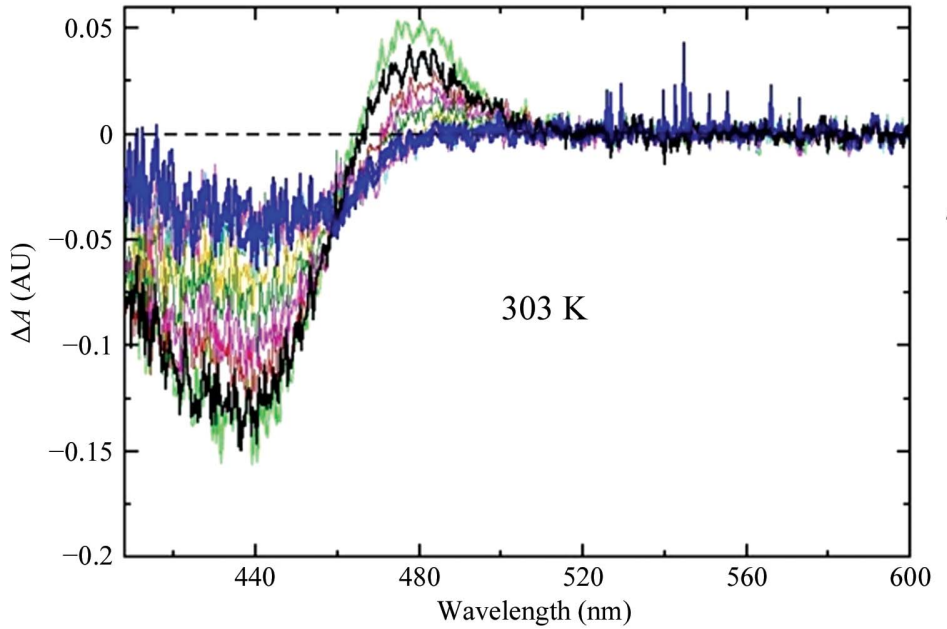

(c)

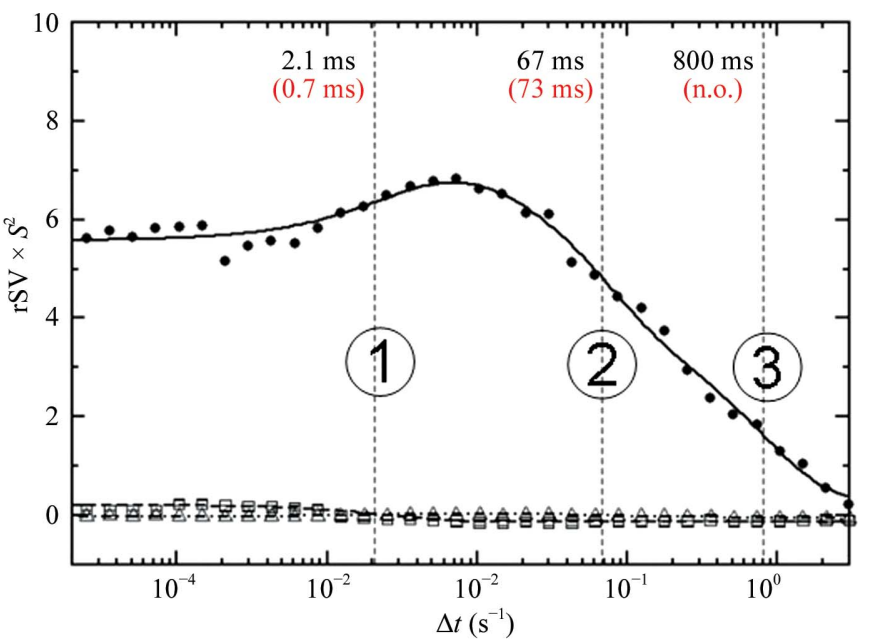

(b)

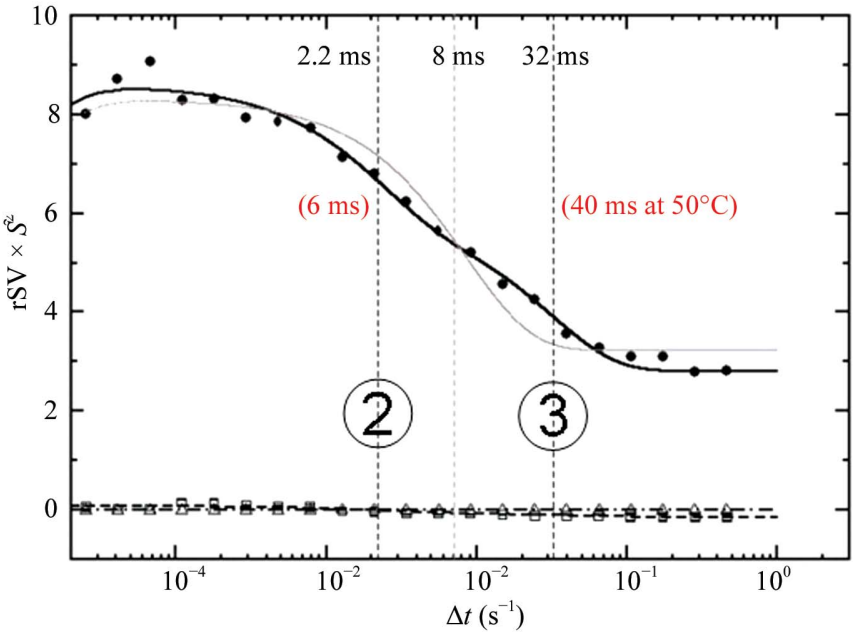

$(d)$

Figure 6

Time-resolved difference absorption spectra of crystalline slurry at $273 \mathrm{~K}(a)$ and $303 \mathrm{~K}(c)$ (black, earliest time point; blue, last time point). ( $b$, $d$ ) Right singular vectors (rSVs) from SVD analysis of the difference spectra. Solid lines, global fit by sums of three exponentials identifying three processes. Processes 1-3 are labeled with their respective relaxation times and are marked with thin vertical lines; relaxation times from crystallography are shown in red brackets (n.o., not observed). The gray line in $(d)$ is the fit of the final relaxation with only one exponential. 
corresponding observable kinetic phases. However, these phases result from a number of underlying interconversions between intermediate states determined by microscopic rate coefficients of the mechanism in Fig. 3. Therefore, the $E_{\mathrm{a}}$ values in Table 2 are not, or are only approximately, meaningful for individual interconversions, and a chemical kinetic mechanism is needed. Within the constraints of the mechanism employed, the TSE allows the separation of entropic and enthalpic contributions to the barriers from the temperature-dependent microscopic rate coefficients (Fig. 1). PYP intermediates occupy minima on the free-energy surface because the chromophore is immersed into a tight hydrogenbonding network. $\Delta H^{\#}$ is positive because for the reaction to occur some of the bonds have to be broken. $\Delta S^{\#}$ reflects the gain or loss of degrees of freedom. If the protein has time to relax, the structure can fluctuate through the substates and the entropy change is positive (Parak et al., 2007). If, however, the protein environment stays rigid, there might be only one well defined narrow path for the transition. In this case $\Delta S^{\#}$ may become negative. By inspecting the structures of the intermediates, the structural reasons for the observed values become clear. However, the structures of the transition states themselves remain unknown because their occupation is minute. With a $\Delta G^{\#}$ of $63 \mathrm{~kJ} \mathrm{~mol}^{-1}$ for the $\mathrm{pB}$ to $\mathrm{pG}$ transition, for example, the probability of catching a molecule on the top of the barrier at $300 \mathrm{~K}$ is only $\exp \left(-\Delta G^{\# / R T}\right)=1.1 \times$ $10^{-11}$. In a macroscopic crystal with about $10^{14}$ molecules, about 1000 molecules are on top of a barrier at any given time. Such low occupancy cannot be detected. However, the structures of the intermediate states that flank the barrier can be determined and the nature of the barrier can be inferred from them. For the $\mathrm{pR}_{2}$ to $\mathrm{pB}$ transition, for example, the chromophore lifts out of a hydrogen-bonding network involving Tyr42, Glu46 and Cys69. $\Delta H^{\#}$ is positive. The chromophore then rotates. On the microsecond time scale protein relaxations are incomplete, as is evident from the absence of extensive features in difference electron-density maps except in the direct vicinity of the chromophore (Figs. $2 a$ and $2 b$ ). Consequently, there is only limited space for this rotation and the entropic contribution to the barrier is slightly negative. The situation is different for the $\mathrm{pB}$ to $\mathrm{pG}$ transition. Once the hydrogen bonds of the pCA head hydroxyl to Arg52 and to one or two water molecules break, the pCA can re-isomerize back to trans. At these longer millisecond to second time scales the protein structure is relaxed, as is obvious from numerous difference electron-density features on protein moieties surrounding the chromophore pocket (Fig. 2c). Once the pCA head is free, it can form a transition state that occupies an enlarged, relaxed chromophore pocket. $\Delta S^{\#}$ becomes positive (Table 1). The entropic contribution helps to accelerate the reaction by lowering the barrier. In solution the PYP structure relaxes even further. For the $\mathrm{pB}$ to $\mathrm{pG}$ transition $\Delta H^{\#}$ is only $10 \mathrm{~kJ} \mathrm{~mol}^{-1}$ at $\mathrm{pH} 3$ and $T \Delta S^{\#}$ is largely negative at $-60 \mathrm{~kJ} \mathrm{~mol}^{-1}$ (Van Brederode et al., 1996). In solution re-isomerization is controlled almost entirely by the entropy and PYP refolds via a transition state which is much more ordered than the $\mathrm{pB}$ intermediate. The structures of both the highly unfolded intermediate and the transition state remain elusive. In the crystal, however, the entropy plays a smaller role because a highly unfolded intermediate (as in solution) apparently does not form.

The PYP photocycle is an excellent model to study macromolecular reactions and to develop new methodologies that can be generalized to the investigation of other proteins and enzymes. By lowering the temperature to below $273 \mathrm{~K}$ reactions slow down so that intermediates such as $\mathrm{I}_{\mathrm{T}}$ (and $\mathrm{I}_{\mathrm{CT}}$ ) that were previously observed only with picosecond timeresolved crystallography (Jung et al., 2013; Schotte et al., 2012) become observable on the nanosecond time scale. Barriers of activation of reactions that occur within biological macromolecules can be determined from time-resolved crystallographic data alone. When pharmaceutically relevant enzymatic reactions are investigated each intermediate structure can be a potential drug target. However, the determination of transient structures in enzymes is more difficult because their reactions are noncyclic or irreversible. Freeenergy landscapes including barriers of activation are decisive for their function. It is therefore desirable to develop methods to rapidly and routinely investigate these reactions on the near-atomic length scale by TR methods at the synchrotron or potentially at the X-ray free-electron laser with microcrystals and nanocrystals (Schmidt, 2013; Aquila et al., 2012; Neutze \& Moffat, 2012) or even in solution (Poon et al., 2013). In solution, as well as in very small crystals, diffusion times are short. This enables rapid reaction initiation by simply mixing the crystals or solution with substrate and injecting the mixture into the X-ray beam (Schmidt, 2013). Photosensitivity has been engineered genetically into protein structures (Möglich et al., 2010), and specially designed and manufactured caged substrates (Goelder \& Givens, 2005) are also used for convenient reaction initiation by laser pulses. These methods, in combination with new and existing powerful X-ray sources, will be available to explore free-energy surfaces and investigate a multitude of enzymatic reactions with five-dimensional crystallography.

\section{Conclusions}

One hundred years after X-ray diffraction was discovered, we show how crystallography can be used to determine barriers of activation in biological macromolecules. Our findings obtained solely from crystallography demonstrate that from 233 to $323 \mathrm{~K}$ all processes in PYP are thermally activated and show approximate Arrhenius behavior. Above $323 \mathrm{~K}$ the reaction surpasses its temperature optimum and slows down again. Meaningful thermodynamic parameters that describe barriers of activation in the PYP photocycle can be extracted and structurally understood.

MS is supported by NSF CAREER grant 0952643 . HI is supported by the Institute for Basic Science (IBS) in the Republic of Korea. Use of BioCARS was supported by the National Institutes of Health, National Institute of General Medical Sciences grant P41GM103543 (formerly National 
Center for Research Resources P41RR007707). The timeresolved setup at Sector 14 was funded in part through a collaboration with Philip Anfinrud (NIH/NIDDK). Use of the Advanced Photon Source, an Office of Science User Facility operated for the US Department of Energy (DOE) Office of Science by Argonne National Laboratory, was supported by the US DOE under Contract No. DE-AC02-06CH11357. This publication was supported by NSF STC 1231306.

\section{References}

Aquila, A. et al. (2012). Opt. Express, 20, 2706-2716.

Austin, R. H., Beeson, K. W., Eisenstein, L., Frauenfelder, H. \& Gunsalus, I. C. (1975). Biochemistry, 14, 5355-5373.

Berman, H. M. et al. (2002). Acta Cryst. D58, 899-907.

Borgstahl, G. E., Williams, D. R. \& Getzoff, E. D. (1995). Biochemistry, 34, 6278-6287.

Borucki, B., Joshi, C. P., Otto, H., Cusanovich, M. A. \& Heyn, M. P. (2006). Biophys. J. 91, 2991-3001.

Borucki, B., Kyndt, J. A., Joshi, C. P., Otto, H., Meyer, T. E., Cusanovich, M. A. \& Heyn, M. P. (2005). Biochemistry, 44, 1365013663.

Brederode, M. E. van, Gensch, T., Hoff, W. D., Hellingwerf, K. J. \& Braslavsky, S. E. (1995). Biophys. J. 68, 1101-1109.

Cornish-Bowden, A. (2012). Fundamentals of Enzyme Kinetics, 4th ed. Weinheim: Wiley-VCH.

Eyring, H. (1935). J. Chem. Phys. 3, 107-115.

Fitzpatrick, A. E., Lincoln, C. N., van Wilderen, L. J. \& van Thor, J. J. (2012). J. Phys. Chem. B, 116, 1077-1088.

Genick, U. K., Borgstahl, G. E., Ng, K., Ren, Z., Pradervand, C., Burke, P. M., Srajer, V., Teng, T.-Y., Schildkamp, W., McRee, D. E., Moffat, K. \& Getzoff, E. D. (1997). Science, 275, 1471-1475.

Gibson, Q. H. (1952). J. Physiol. 117, 49P-50P.

Goelder, M. \& Givens, R. (2005). Dynamic Studies in Biology: Phototriggers, Photoswitches and Caged Biomolecules. Weinheim: Wiley-VCH.

Graber, T. et al. (2011). J. Synchrotron Rad. 18, 658-670.

Groenhof, G., Bouxin-Cademartory, M., Hess, B., de Visser, S. P., Berendsen, H. J. C., Olivucci, M., Mark, A. E. \& Robb, M. A. (2004). J. Am. Chem. Soc. 126, 4228-4233.

Hanggi, P., Talkner, P. \& Borkovec, M. (1990). Rev. Mod. Phys. 62, 251-341.

Ihee, H., Rajagopal, S., Srajer, V., Pahl, R., Anderson, S., Schmidt, M., Schotte, F., Anfinrud, P. A., Wulff, M. \& Moffat, K. (2005). Proc. Natl Acad. Sci. USA, 102, 7145-7150.

Jung, Y. O., Lee, J. H., Kim, J., Schmidt, M., Moffat, K., Šrajer, V. \& Ihee, H. (2013). Nature Chem. 5, 212-220.

Kim, T. W., Lee, J. H., Choi, J., Kim, K. H., van Wilderen, L. J., Guerin, L., Kim, Y., Jung, Y. O., Yang, C., Kim, J., Wulff, M., van Thor, J. J. \& Ihee, H. (2012). J. Am. Chem. Soc. 134, 3145-3153.

Martin, J. L., Migus, A., Poyart, C., Lecarpentier, Y., Astier, R. \& Antonetti, A. (1983). Proc. Natl Acad. Sci. USA, 80, 173-177.
Meyer, T. E., Devanathan, S., Woo, T., Getzoff, E. D., Tollin, G. \& Cusanovich, M. A. (2003). Biochemistry, 42, 3319-3325.

Moffat, K. (1989). Annu. Rev. Biophys. Biophys. Chem. 18, 309-332. Moffat, K. (2001). Chem. Rev. 101, 1569-1581.

Möglich, A., Ayers, R. A. \& Moffat, K. (2010). J. Mol. Biol. 400, 477-486.

Neutze, R. \& Moffat, K. (2012). Curr. Opin. Struct. Biol. 22, 651-659.

Ng, K., Getzoff, E. D. \& Moffat, K. (1995). Biochemistry, 34, 879-890.

Parak, F. G., Achterhold, K., Croci, S. \& Schmidt, M. (2007). J. Biol. Phys. 33, 371-387.

Poon, H.-C., Schmidt, M. \& Saldin, D. K. (2013). Adv. Condens. Matter Phys. 2013, 750371.

Purwar, N., Tenboer, J., Tripathi, S. \& Schmidt, M. (2013). Int. J. Mol. Sci. 14, 18881-18898.

Rajagopal, S., Anderson, S., Srajer, V., Schmidt, M., Pahl, R. \& Moffat, K. (2005). Structure, 13, 55-63.

Schmidt, M. (2008). Ultrashort Laser Pulses in Medicine and Biology, edited by M. Braun, P. Gilch \& W. Zinth, pp. 201-237. Berlin: Springer.

Schmidt, M. (2013). Adv. Condens. Matter Phys. 2013, 167276

Schmidt, M., Graber, T., Henning, R. \& Srajer, V. (2010). Acta Cryst. A66, 198-206.

Schmidt, M., Ihee, H., Pahl, R. \& Šrajer, V. (2005). Methods Mol. Biol. 305, 115-154.

Schmidt, M., Nienhaus, K., Pahl, R., Krasselt, A., Anderson, S., Parak, F., Nienhaus, G. U. \& Šrajer, V. (2005). Proc. Natl Acad. Sci. USA, 102, 11704-11709.

Schmidt, M., Pahl, R., Srajer, V., Anderson, S., Ren, Z., Ihee, H., Rajagopal, S. \& Moffat, K. (2004). Proc. Natl Acad. Sci. USA, 101, 4799-4804.

Schmidt, M., Rajagopal, S., Ren, Z. \& Moffat, K. (2003). Biophys. J. 84, 2112-2129.

Schmidt, M., Šrajer, V., Purwar, N. \& Tripathi, S. (2012). J. Synchrotron Rad. 19, 264-273.

Schotte, F., Cho, H. S., Kaila, V. R., Kamikubo, H., Dashdorj, N., Henry, E. R., Graber, T. J., Henning, R., Wulff, M., Hummer, G., Kataoka, M. \& Anfinrud, P. A. (2012). Proc. Natl Acad. Sci. USA, 109, 19256-19261.

Šrajer, V., Teng, T., Ursby, T., Pradervand, C., Ren, Z., Adachi, S., Schildkamp, W., Bourgeois, D., Wulff, M. \& Moffat, K. (1996). Science, 274, 1726-1729.

Steinfeld, J. I., Francisco, J. S. \& Hase, W. L. (1985). Chemical Kinetics and Dynamics, 2nd ed. New Jersey: Prentice Hall.

Stokkum, I. H. van, Larsen, D. S. \& van Grondelle, R. (2004). Biochim. Biophys. Acta, 1657, 82-104.

Takeshita, K., Imamoto, Y., Kataoka, M., Tokunaga, F. \& Terazima, M. (2002). Biochemistry, 41, 3037-3048.

Tripathi, S., Srajer, V., Purwar, N., Henning, R. \& Schmidt, M. (2012). Biophys. J. 102, 325-332.

Van Brederode, M. E., Hoff, W. D., Van Stokkum, I. H., Groot, M. L. \& Hellingwerf, K. J. (1996). Biophys. J. 71, 365-380.

Yeremenko, S., van Stokkum, I. H., Moffat, K. \& Hellingwerf, K. J. (2006). Biophys. J. 90, 4224-4235. 